Read all three of our prestigious publications, each offering high-quality content to keep you informed with the latest developments in the field.
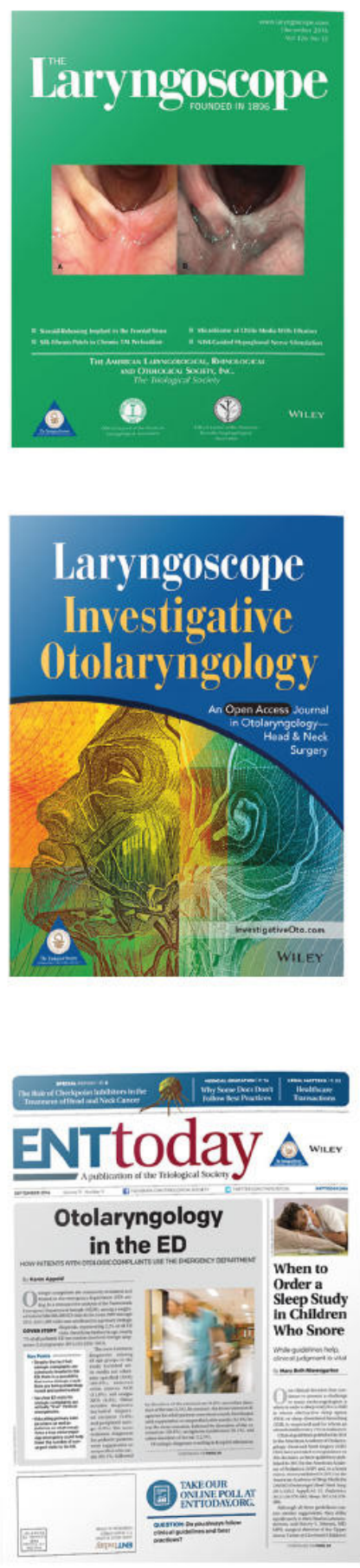

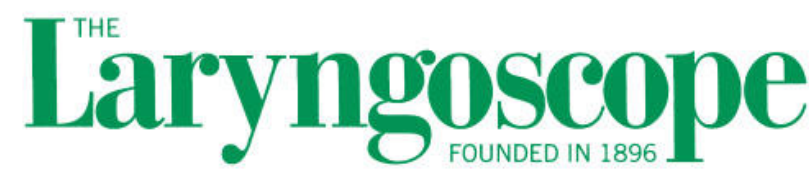

Editor-in-Chief: Michael G. Stewart, MD, MPH

The leading source for information in head and neck disorders.

\section{Laryngoscope.com}

Laryngoscope

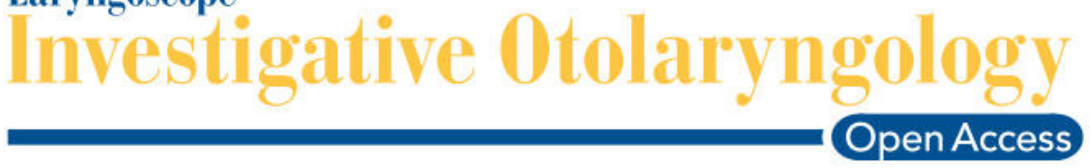

Editor-in-Chief: D. Bradley Welling, MD, PhD, FACS

Rapid dissemination of the science and practice of otolaryngology-head and neck surgery.

InvestigativeOto.com

\section{ENTtoday}

Editor-in-Chief: Alexander Chiu, MD

Must-have timely information that Otolaryngologisthead and neck surgeons can use in daily practice.

\section{Enttoday.org}




\title{
Long-term Neurological Morbidity Following Endoscopic Transnasal Resection of Juvenile Angiofibroma
}

\author{
Davide Mattavelli, MD (1); Vittorio Rampinelli, MD (1); Marco Ferrari, MD; Alberto Schreiber, MD, PhD; \\ Bruno Guarneri, MD; Piero Nicolai, MD
}

\begin{abstract}
Objectives/Hypothesis: Although transnasal endoscopic resection (TER) of juvenile angiofibroma (JA) is unquestionably less invasive than traditional external approaches, several endonasal and neurovascular structures are sacrificed during the procedure. The aim of this study was to evaluate long-term neurological morbidity and related quality of life following TER of JA.

Study Design: Retrospective cohort study.

Methods: All patients who underwent TER for JA at the Unit of Otorhinolaryngology-Head and Neck Surgery of the University of Brescia from 1994 to 2016 were contacted to know their availability to undergo a battery of tests aimed to assess lacrimal secretion (Schirmer test), impairment of sensitive nerves (electrophysiological threshold test), and impact on quality of life of tearing reduction and sensitivity impairment with the Ocular Surface Disease Index (OSDI) and visual analogue scale (VAS) (0-10), respectively.

Results: Thirteen patients were included. Mean follow-up was 77 months (range, 19-156 months). The median Schirmer test value was $5.5 \mathrm{~mm}$ and $28.5 \mathrm{~mm}$ for the treated and untreated sides, respectively $(P=.003)$. Analysis of sensitivity revealed significant impairment only in the hard palate. The mean OSDI score was 6.8 (normal). The mean values of the VAS scores for hard palate, buccal mucosa, gum, and premaxillary skin were 1.7, 1.7, 1.2, and 2.3, respectively.

Conclusions: TER for JA can result in objective reduction of lacrimal secretion and sensitivity impairment; nevertheless, their impact on quality of life is negligible. The predictable neurological morbidity of TER should be discussed during preoperative counseling.

Key Words: Endoscopic endonasal surgery, juvenile angiofibroma, neurological morbidity, quality of life, dry eye syndrome.
\end{abstract}

Level of Evidence: 4

Laryngoscope, 00:1-5, 2018

\section{INTRODUCTION}

In the last decades, transnasal endoscopic resection (TER) of juvenile angiofibroma (JA) has become the mainstay in surgical management of this disease. The results of a systematic review published in 2013 demonstrated that TER is associated with lower intraoperative blood loss and a similar recurrence rate compared to open resections. ${ }^{1}$ Moreover, TER is considered significantly less invasive than open techniques in view of the absence of transfacial and/or transcranial incisions/osteotomies. ${ }^{1,2}$ However, during an endoscopic approach to the JA, a number of neurovascular structures are encountered, manipulated, or sacrificed, and data on long-term,

From the Unit of Otorhinolaryngology-Head and Neck Surgery (D.M., V.R., M.F., A.S., P.N.); and Unit of Electrophysiology (B.G.), University of Brescia, Brescia, Italy

Editor's Note: This Manuscript was accepted for publication July 5, 2018.

The authors have no funding, financial relationships, or conflicts of interest to disclose.

Send correspondence to Davide Mattavelli, MD, Department of Otorhinolaryngology, University of Brescia, Piazzale Spedali Civili 1, 25123 Brescia, Italy. E-mail: d.mattavelli@unibs.it

DOI: 10.1002/lary.27466 postsurgical neurological morbidity and quality of life are lacking.

According to some authors, ${ }^{3} \mathrm{JA}$ is thought to arise from nonregressed remnants of the first branchial artery, which is located in the area of the vidian canal. Therefore, the base of the pterygoid process, where the vidian canal is located, is considered a hotspot area, which needs to be exposed and drilled regardless of the extent of the lesion to achieve radical resection. Consequently, the vidian, descending palatine, and sphenopalatine nerves are almost invariably sectioned. Furthermore, several sensitive nerves (infraorbital, posterior superior alveolar, and buccal nerves) may be at high risk of damage, especially when the lesion extends into the infratemporal fossa. Therefore, impairment of orofacial sensitivity and deficit of ipsilateral lacrimation should be expected as a consequence of injury to the trigeminal system and vidian nerve, respectively.

The aim of the present study was to explore the long-term neurological morbidity of TER for JA. In particular, impairment of orofacial sensitivity and lacrimal secretion was objectively assessed by electrophysiological and Schirmer tests, respectively. Moreover, the impact on quality of life was investigated with dedicated questionnaires. 


\section{MATERIALS AND METHODS}

The study was conducted according to the principles of the revised Declaration of Helsinki, in compliance with Good Clinical Practice and ethical standards, and was approved by the local ethics committee (Comitato Etico Provinciale di Brescia).

Data of all patients undergoing TER for JA at the Unit of Otorhinolaryngology-Head and Neck Surgery of the University of Brescia (Brescia, Italy) from January 1994 to June 2016 were retrieved from a dedicated database. Patients with neurologic disease, mental illness, autoimmune disorder, previous surgical procedures in the anatomic regions of interest (including dental procedures with potential impairment of the alveolar sensitivity), or a follow-up $<12$ months were excluded. All eligible patients were contacted by telephone, the study design was explained, and the patients were asked to participate.

\section{Surgical Technique}

The surgical technique for TER of JA has been previously reported by our group in numerous publications. ${ }^{4-6}$ The procedure usually starts with posterior septectomy and removal of the sphenoidal rostrum and endoscopic medial maxillectomy (EMM), which is modulated depending on the lateral extension of the JA in the infratemporal fossa. ${ }^{7}$ Total ethmoidectomy, opening of the descending palatine canal, and removal of the medial portion of the posterior maxillary wall allows exposure of the JA behind the periosteum of the pterygopalatine and infratemporal fossae. Identification and ligature of the maxillary artery is performed. All the possible components of the lesion extending into the infratemporal fossa, inferior orbital fissure, foramen rotundum, sphenoid sinus, vidian canal, pterygoid root, and nasopharynx are gently pulled and dissected from surrounding tissues. The base of the pterygoid, being the epicenter of the lesion, is exposed and drilled in the area of the vidian canal, well beyond the apparent area of bone erosion. The same approach on the bone is adopted whenever the JA extends into the sphenoidal greater wing, the pterygoid plates, or the floor of the sphenoid sinus.

\section{Analysis of Lacrimal Function}

Lacrimal secretion was assessed bilaterally with the Schirmer test (Alfa Intes, Casoria, Italy). The impact of lacrimal impairment on quality of life was investigated using a validated questionnaire called the Ocular Surface Disease Index (OSDI). ${ }^{8}$

\section{Analysis of Sensitivity}

The area innervated by the sensitive nerves at higher risk of damage was topographically analysed and arbitrarily divided into subareas (Fig. 1).

The hard palate (mostly innervated by the descending palatine nerve through the greater palatine nerve) was divided into six subunits (three per side), defined by two coronal planes joining the canines and first molars on each side, and the midline (areas A, B, and C in Fig. 1). The buccal mucosa (mostly innervated by the buccal nerve) was divided into four subunits defined by the axial plane passing through the labial commissure and the coronal plane passing through the first molar (areas D, E, F, and $G$ in Fig. 1). The sensitivity of the superior alveolar ridge (mostly innervated by the anterior and posterior superior alveolar nerves) was measured at the level of the gum overlying the alveolus of the canine and first molar (3 and 6 in Fig. 1). The sensitivity of the premaxillary skin (mostly innervated by the infraorbital nerve) was studied $1 \mathrm{~cm}$ below the infraorbital foramen (area PMS in Fig. 1).

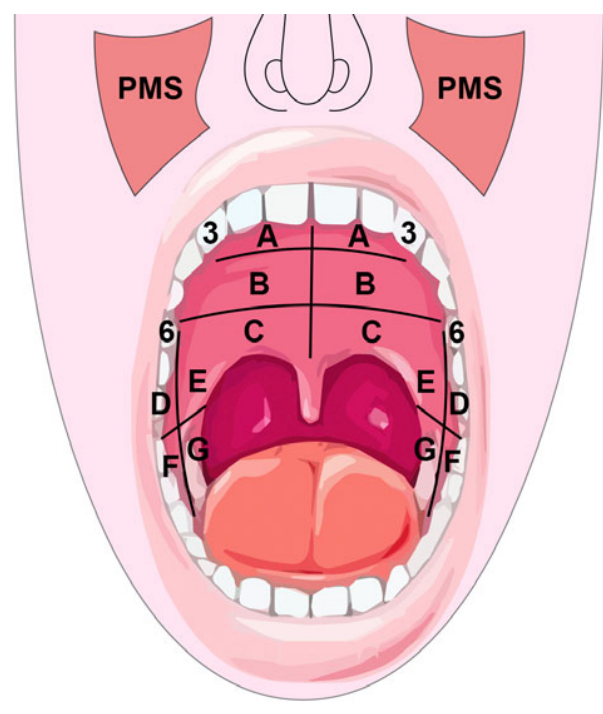

Fig. 1. Schematic representation of mucosal and skin areas tested with the electrophysiological method. 3 = canine (alveolar mucosa); 6 = first molar (alveolar mucosa); $A-C=$ subunits of the palate; $D-G$ = subunits of the buccal mucosa; PMS = premaxillary skin. [Color figure can be viewed at www.laryngoscope.com.]

The sensitivity of each area was measured using an electrophysiological threshold test, which consisted of a pulsating electric stimulation applied with a dipole positioned in contact with the mucosa or skin of each area (Nicolet Viking Quest; Natus Medical Neurology, Middleton, WI). The intensity of the stimulation was gradually increased by $1 \mathrm{mV}$ per stimulation starting from $0 \mathrm{mV}$, with a frequency of the stimulus of $2 \mathrm{~Hz}$, until the pulsating stimulation was perceived by the patient. The threshold value was accordingly recorded. The test was performed in every subunit on both sides, and the untreated side was considered as the reference of normality.

The visual analogue scale (VAS) score (from $0=$ no detrimental impact to $10=$ maximal negative impact) was used to quantify changes in quality of life related to any possible sensitivity impairment in each anatomical area of interest (hard palate, alveolar ridge, buccal mucosa, and premaxillary skin).

\section{Statistical Analysis}

Statistical analysis was performed using the XLSTAT addon for Microsoft Excel version 2015.4.01 (Addinsoft SARL, Paris, France). Descriptive statistics were used for clinical records, lacrimal function analysis, and sensitivity analysis. Lacrimal secretion and thresholds of sensitivity were compared with the Wilcoxon test. A $P$ value $<.05$ was considered statistically significant.

\section{RESULTS}

Seventy-eight patients were retrieved from the database; 51 were eligible and were contacted by telephone. Thirteen patients (all males) agreed to participate in the study and signed an informed consent. The mean age at the time of surgery was 17 years (median: 16 years, range: $10-35$ years), and the mean follow-up after surgery was 77 months (median: 70 months, range: 19-156 months). Patients were distributed according to the most widely used staging systems, as follows: Andrews et al. ${ }^{9}$ : 
one $(7.7 \%)$ stage I, six $(46.2 \%)$ stage II, one $(7.7 \%)$ stage IIIa, four $(30.8 \%)$ stage IIIb, and one $(7.7 \%)$ stage IVb; Radkowski et al. ${ }^{10}$ : one $(7.7 \%)$ stage Ia, one $(7.7 \%)$ stage IIa, three $(23.1 \%)$ stage IIb, one $(7.7 \%)$ stage IIc, six (46.2\%) stage IIIa, and one $(7.7 \%)$ stage IIIb; Snyderman et al. ${ }^{11}$ : seven $(53.8 \%)$ stage I, one $(7.7 \%)$ stage II, two (15.5\%) stage III, two (15.4\%) stage IV, and one $(7.7 \%)$ stage V. Twelve of the $13(92.3 \%)$ patients underwent preoperative endovascular embolization.

In all patients, TER encompassed the dissection of the pterygopalatine fossa, section of vidian, descending palatine, and sphenopalatine nerves, and drilling of the base of the pterygoid process. The infraorbital, posterior superior alveolar, and buccal nerves were never sacrificed intentionally. Twelve $(92.3 \%)$ patients underwent a type B EMM, whereas in the remainder, a type C EMM was performed. ${ }^{7}$ Type D EMM (i.e., Sturmann-Canfield procedure) was never performed.

\section{Analysis of Lacrimal Function}

The median Schirmer test value was 5.0 mm (mean: $6.6 \mathrm{~mm}$ ) and $27.0 \mathrm{~mm}$ (mean: $24.8 \mathrm{~mm}$ ) for the treated and untreated side, respectively. This difference (median: $21.0 \mathrm{~mm}$, mean: $19.3 \mathrm{~mm})$ was significant $(P=.003)$ (Fig. 2). The mean OSDI score was 6.8, which is considered normal. ${ }^{8}$

\section{Analysis of Sensitivity}

Data on the threshold of sensitivity of each area analyzed are summarized in Table I and Figure 3. Buccal mucosa, alveolar ridge mucosa, and premaxillary skin did not show significant differences between the two sides.

At the level of the hard palate, a significant difference was found for the anterior $(P=.003)$ and middle $(P=.006)$ areas, whereas the difference was close to significance $(P=.054)$ for the posterior portion. The difference between the two sides constantly increased from posterior to anterior (Table I, Fig. 3).

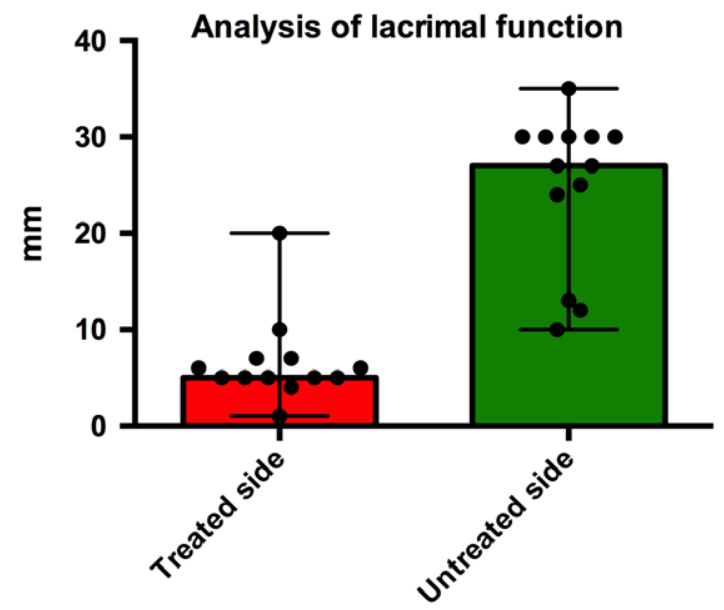

Fig. 2. Histogram plot showing the results of the lacrimal function analysis. Black dots mark single measurements and black bars show the range of values. [Color figure can be viewed at www.laryn goscope.com.]
TABLE I.

Median Values of the Threshold of Sensitivity for Each Area.

\begin{tabular}{lrrr}
\hline Area & Treated Side $(\mathrm{mV})$ & Untreated Side $(\mathrm{mV})$ & $P$ Value \\
\hline A & $12.0(13.4)$ & $9.0(9.5)$ & .003 \\
B & $16.0(14.8)$ & $12.0(11.3)$ & .006 \\
C & $14.0(13.7)$ & $12.0(10.9)$ & .054 \\
D & $10.0(12.0)$ & $8.0(9.0)$ & .180 \\
E & $10.0(12.2)$ & $11.0(10.6)$ & .608 \\
F & $8.0(9.2)$ & $8.0(9.1)$ & .918 \\
G & $10.0(10.5)$ & $9.0(9.8)$ & .832 \\
3 & $7.0(8.1)$ & $7.0(7.6)$ & .426 \\
6 & $10.0(10.2)$ & $8.0(8.4)$ & .326 \\
PMS & $22.0(25.0)$ & $24.0(22.6)$ & .300 \\
\hline \hline
\end{tabular}

Mean values are reported in parentheses.

3 = canine (alveolar mucosa); 6 = first molar (alveolar mucosa); A-C = subunits of the palate; D-G = subunits of the buccal mucosa; PMS = premaxillary skin.

The mean values of the VAS score of the hard palate, alveolar ridge, buccal mucosa, and premaxillary skin were $1.7,1.7,1.2$, and 2.3 , respectively.

\section{DISCUSSION}

The aim of the present study was to evaluate the long-term neurological morbidity of TER for JA in a homogeneous cohort of patients treated in a single referral center. Morbidity was explored in terms of sensitivity impairment and reduction of lacrimal secretion, according to the neural structures inevitably sacrificed during the procedure. Objective, measurable variables were analyzed, and a subjective questionnaire was used. Our results demonstrate that a difference between treated and untreated sides is objectively detectable, but it does not impact the long-term quality of life of patients.

TER for JA is largely validated in the literature. ${ }^{1,2,4,5}$ When performed, the procedure must follow well-defined surgical steps and concepts: adequate exposure of the JA, coagulation and section of the main afferent vessels, exposure and drilling of the basisphenoid and vidian canal, as it is the main hotspot for persistence. ${ }^{5,12}$ Accordingly, the vidian, descending palatine, and sphenopalatine nerves are unavoidably sectioned in almost all cases. Furthermore, the infraorbital, posterior superior alveolar, and buccal nerves are frequently and inadvertently manipulated and stretched during dissection of the JA in the pterygopalatine and infratemporal fossae. Therefore, impairment of orofacial sensitivity and lacrimation should be expected as sequelae of the endoscopic procedure. To the best of our knowledge, sensitivity impairment and dry eye syndrome after TER for JA have never been systematically investigated.

Lacrimal secretion was significantly reduced in all patients on the operated side (Fig. 2). However, the impact on quality of life based on the results of the OSDI was negligible. Interestingly, our experience parallels that reported in the literature on functional outcomes of vidian neurectomy for incoercible rhinitis. A recent 

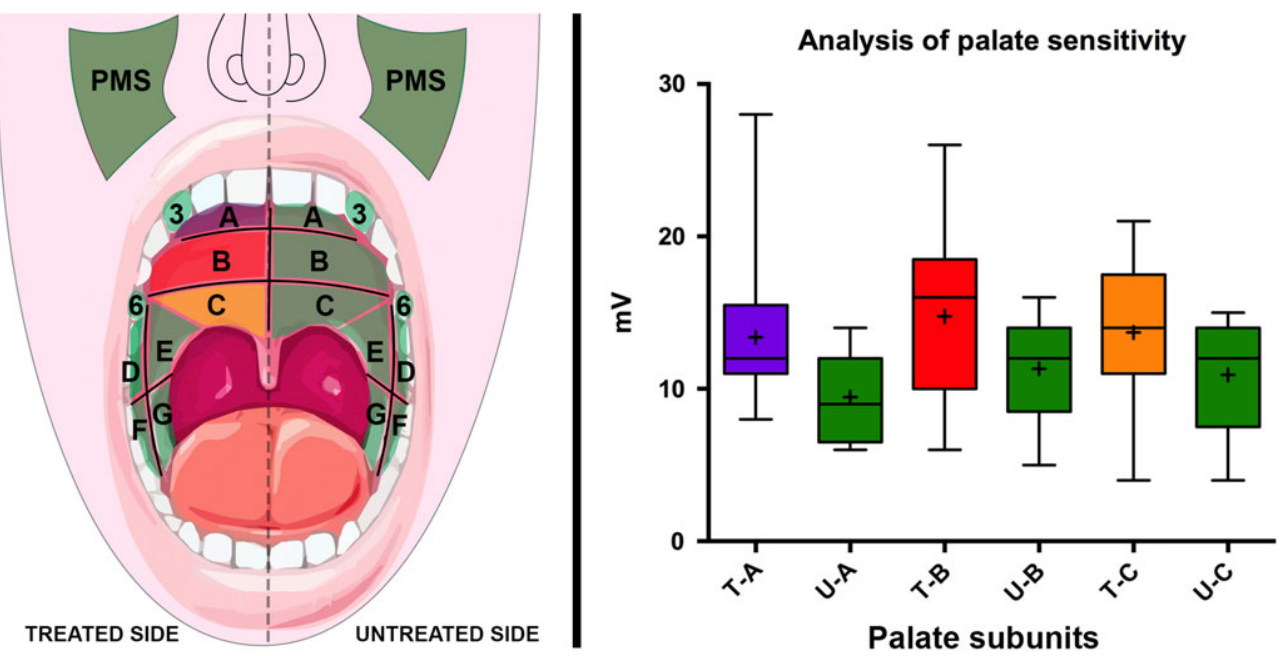

Fig. 3. Schematic representation of sensitivity thresholds and box plots comparing treated and untreated sides. On the treated side, sensitivity impairment was observed only in palate subunits (yellow [C], red $[B]$, and purple $[A]$ areas), whereas it was preserved in the remainders (green areas). The box plot summarizes the median (black bar within the box), mean (black + ), interquartile interval (black box boundaries), and range (black bars) of sensitivity thresholds. $\mathrm{PMS}=$ premaxillary skin; $\mathrm{T}-\mathrm{A}=$ area $\mathrm{A}$, treated side; $\mathrm{T}-\mathrm{B}=$ area $\mathrm{B}$, treated side; $\mathrm{T}-\mathrm{C}=$ area $\mathrm{C}$, treated side; $\mathrm{U}-$ $A=$ area $A$, untreated side; $U-B=$ area $B$, untreated side; $\mathrm{U}-\mathrm{C}=$ area $\mathrm{C}$, untreated side. [Color figure can be viewed at www.laryngoscope.com.]

review analyzing 16 studies reported a dry eye condition in $23.96 \%(276 / 1,152)$ of patients. ${ }^{13}$ A subanalysis limited to eight studies, also addressing the duration of dry eye symptoms, found that they were temporary in $96.46 \%$ of patients $(218 / 226)$ and spontaneously resolved within 6 months. ${ }^{13}$

The analysis of sensitivity impairment provided more complex results. At the level of the buccal, alveolar ridge, and premaxillary regions, the threshold of sensitivity between the treated and untreated sides was comparable, which suggests that the buccal, superior alveolar, and infraorbital nerves, respectively, were preserved. These data could be at least partially explained by the expansive and noninvasive pattern of growth of the JA, which allows for smooth and conservative dissection of the lesion from surrounding tissues.

The anterior and middle portion of the hard palate on the treated side showed a significant deficit in sensitivity compared to the untreated side, with increasing sensitivity impairment from posterior to anterior (Fig. 3).

When a sensory nerve is damaged, reinnervation of its territory usually comes from adjacent areas. In our model, anesthesia of the hemipalate after section of the descending palatine nerves could potentially be compensated by the contralateral hemipalate or the ipsilateral superior alveolar plexus, via the incisive nerve. Possible compensation from the nasopalatine nerve is, however, prevented by the proximal section of the sphenopalatine nerve. Our results support the existence of reinnervation from the contralateral areas following a centrifugal direction with respect to the central nervous system. ${ }^{14,15}$ This mechanism would explain the better compensation in the posterior portion (closer to the brain) rather than in the middle and anterior areas. Conversely, the contribution provided by the superior alveolar plexus is apparently minimal, because the anterior region, which should benefit the most, is where the sensory deficit is more evident.
The potential contribution of endovascular embolization to the sensitivity impairment is not assessable in our series, because all of the patients except one underwent this preoperative procedure. Nevertheless, some considerations can be done. All of the nerves we analyzed were vascularized by the network of the maxillary artery, thus being theoretically all at risk of ischemic damage during embolization. However, the only permanent sensitive deficit we observed was limited to the territory of the descending palatine nerve, which was invariably sectioned during surgery. Therefore, we can speculate that the overall impact of embolization on long-term peripheral neurological morbidity is likely negligible.

Similar to lacrimal dysfunction, objective and detectable impairment in sensitivity of the mucosa of the hard palate was not reflected in a worsening of the quality of life. The values of the VAS scale were low, indicating minimal impact on quality of life for all the assessed subunits.

The strong points of our study are the strict inclusion criteria and the in-depth analysis of expected neurological sequelae. In particular, the use of both objective measurements and subjective questionnaires enabled us to identify a discrepancy between the detectable longterm morbidity (reduction of lacrimal secretion and hard palate sensitivity impairment) and the negligible impact that they have on the quality of life of patients.

These findings further confirm the validity of TER for JA treatment in terms of functional outcomes. The predictable morbidity of the procedure should be thoroughly discussed during preoperative counseling; however, the attention of the patient and parents should be focused on the excellent long-term results for quality of life.

The main limitations of the study are its retrospective nature and the small series presented. Restrictive inclusion criteria and the rarity of the disease, as well as the invasiveness of the analysis performed can justify the 
limited number of cases recruited for the study. Nevertheless, the statistical significance obtained in different tests supports the validity of our analysis.

\section{CONCLUSION}

TER of JA entails manipulation and sacrifice of different neurovascular structures, with consequential and objectively measurable impairment of their function on the treated side. The negligible impact on long-term quality of life is an additional argument to consider TER as the mainstay of treatment for JA. Predictable morbidity of the procedure must still be discussed during preoperative counseling.

\section{BIBLIOGRAPHY}

1. Boghani Z, Husain Q, Kanumuri VV, et al. Juvenile nasopharyngeal angiofibroma: a systematic review and comparison of endoscopic, endoscopic-assisted, and open resection in 1047 cases. Laryngoscope 2013; 123:859-869.

2. Langdon C, Herman P, Verillaud B, et al. Expanded endoscopic endonasal surgery for advanced stage juvenile angiofibromas: a retrospective multi-center study. Rhinology 2016;54:239-246.
3. Schick B, Plinkert PK, Prescher A. Aetiology of Angiofibromas: reflection on their specific vascular component. Laryngorhinootologie 2002;81:280-284.

4. Nicolai P, Villaret AB, Farina D, et al. Endoscopic surgery for juvenile angiofibroma: a critical review of indications after 46 cases. Am J Rhinol Allergy 2010;24:e67-e72.

5. Nicolai P, Schreiber A, Bolzoni Villaret A. Juvenile angiofibroma: evolution of management. Int J Pediatr 2012;2012:412545.

6. Schreiber A, Safadi A, Ravanelli M, et al. Juvenile angiofibroma. In: De Souza C, Chaturvedi P, Swift AC, et al., eds. Otorhinolaryngology Head and Neck Surgery. 2nd ed. New Delhi, India: Jaypee Brothers; 2017.

7. Schreiber A, Ferrari M, Rampinelli V, et al. Modular endoscopic medial maxillectomies: quantitative analysis of surgical exposure in a preclinical setting. World Neurosurg 2017;100:44-55.

8. Schiffman RM, Christianson MD, Jacobsen G, et al. Reliability and validity of the Ocular Surface Disease Index. Arch Ophthalmol 2000;118:615-621.

9. Andrews JC, Fisch U, Valavanis A, et al. The surgical management of extensive nasopharyngeal angiofibromas with the infratemporal fossa approach. Laryngoscope 1989;99:429-437.

10. Radkowski D, McGill T, Healy GB, et al. Angiofibroma. Changes in staging and treatment. Arch Otolaryngol Head Neck Surg 1996;122: $122-129$.

11. Snyderman CH, Pant H, Carrau RL, Gardner P. A new endoscopic staging system for angiofibromas. Arch Otolaryngol Head Neck Surg 2010;136 $588-594$.

12. Howard DJ, Lloyd G, Lund V. Recurrence and its avoidance in juvenile angiofibroma. Laryngoscope 2001;111:1509-1511.

13. Marshak T, Yun WK, Hazout C, et al. A systematic review of the evidence base for vidian neurectomy in managing rhinitis. J Laryngol Otol 2016; 130(suppl 4):S7-S28

14. Burnett MG, Zager EL. Pathophysiology of peripheral nerve injury: a brief review. Neurosurg Focus 2004;16:E1.

15. Menorca RM, Fussell TS, Elfar JC. Nerve physiology: mechanisms of injury and recovery. Hand Clin 2013;29:317-330. 